Yiming Long

\title{
Multiplicity and stability of closed geodesics on Finsler 2-spheres
}

\author{
Dedicated to Professor Antonio Ambrosetti on his 60th birthday
}

Received September 7, 2005 and in revised form November 1, 2005

\begin{abstract}
A survey of recent progress on the multiplicity and stability problems for closed geodesics on Finsler 2-spheres is given.
\end{abstract}

Keywords. Closed geodesics, Finsler metric, 2-sphere

It is well known that the geodesic, i.e., the shortest curve, connecting two prescribed points in the Euclidean plane is the line segment connecting them. But the geodesic, especially the closed geodesic, problem on the earth is very difficult. In fact, the closed geodesic problem is a very important subject in both dynamical systems and differential geometry, and has stimulated many creative ideas and new developments in mathematics. For closed geodesics on spheres with Riemannian or Finsler structures, modern mathematical studies can be traced back at least to the work of J. Hadamard, H. Poincaré, G. D. Birkhoff, M. Morse, L. Lyusternik, L. Schnirelmann, and many other famous mathematicians. In this short survey, I can only refer to some of the vast literature which is related to closed geodesics on 2-spheres and to our current interests. This paper is organized as follows: §1: A partial and certainly incomplete history of the studies of closed geodesics, mainly on 2-spheres. §2: The multiplicity result obtained by Victor Bangert and the author, and the stability result obtained by Wei Wang and the author for closed geodesics on Finsler 2-spheres. §3: Main ideas in the proof of the multiplicity theorem of V. Bangert and the author. $\S 4$ : Open problems.

\section{A partial history of closed geodesics}

First we introduce the concept of Finsler and Riemannian metrics on manifolds.

Definition 1.1 (cf. [BCS], [She] $)$. Let $M$ be a finite-dimensional manifold. A function $F: T M \rightarrow[0, \infty)$ is a Finsler metric if it satisfies

Research partially supported by the 973 Program of MOST, NSFC, Yangzi River Professorship, MCME, SRFDP, LPMC of MOE of China, S. S. Chern Foundation, and Nankai University.

Y. Long: Chern Institute of Mathematics and LPMC, Nankai University, Tianjin 300071, P.R. China; e-mail: longym@ nankai.edu.cn

Mathematics Subject Classification (2000): 53C22, 58E10, 58E05 
(F1) $F$ is $C^{\infty}$ on $T M \backslash\{0\}$,

(F2) $F(x, \lambda y)=\lambda F(x, y)$ for all $y \in T_{x} M, x \in M$, and $\lambda>0$,

(F3) for every $y \in T_{x} M \backslash\{0\}$, the quadratic form

$$
\left.g_{x, y}(u, v) \equiv \frac{1}{2} \frac{\partial^{2}}{\partial s \partial t} F^{2}(x, y+s u+t v)\right|_{t=s=0}, \quad \forall u, v \in T_{x} M,
$$

is positive definite.

In this case, $(M, F)$ is called a Finsler manifold.

$F$ is reversible if $F(x,-y)=F(x, y)$ for all $y \in T_{x} M$ and $x \in M$, and Riemannian if $F(x, y)^{2}=\frac{1}{2} G(x) y \cdot y$ for some symmetric positive definite matrix function $G(x) \in$ $\mathrm{GL}\left(T_{x} M\right)$ depending on $x \in M$ smoothly. We denote by $\mathcal{F}(M)$ and $\mathcal{R}(M)$ the sets of all Finsler and all Riemannian metrics on $M$ respectively.

Note that one of the major differences between Riemannian and Finsler metrics is the irreversibility in the condition (F2). For a closed geodesic $c$ in a Finsler manifold $(M, F)$, its inverse curve $c^{-1}$ defined by $c^{-1}(t)=c(1-t)$ may not be a geodesic. If it is, it is usually viewed as a closed geodesic different from $c$.

For any closed curve $f: S^{1} \rightarrow M$ on a Finsler manifold $(M, F)$ or a Riemannian manifold $(M, g)$, the group $S^{1}$ or $O(2)$ acts on $f$ by $\theta \cdot f(t)=f(t+\theta)$ for every $\theta \in S^{1}$, respectively by $(\varepsilon, \theta) \cdot f(t)=f(\varepsilon t+\theta)$ for every $(\varepsilon, \theta) \in\{ \pm 1\} \times S^{1} \cong O(2)$. For a closed geodesic $c$, its $m$-th iterate is defined by $c^{m}(t)=c(m t)$. A closed geodesic is prime if it is not the $m$-th iterate of any other closed geodesics for any $m \geq 2$. Two prime closed geodesics $c_{1}$ and $c_{2}$ on a Finsler manifold $(M, F)$ (or Riemannian manifold $(M, g)$ ) are distinct if they do not differ by an $S^{1}$-action (or $O(2)$-action). We denote the set of all distinct prime closed geodesics on a Finsler manifold by $\mathrm{CG}(M, F)$, and similarly by $\mathrm{CG}(M, g)$ for Riemannian manifolds.

It is a longstanding conjecture that there exist infinitely many distinct prime closed geodesics on every compact Riemannian manifold (cf. Problem 81 in [Yau]). J. Hadamard in 1898 and H. Poincaré in 1905 studied closed geodesics on convex surfaces (cf. [Had] and [Poi] $)$. Then G. D. Birkhoff proved the following remarkable result:

Theorem 1.2 (G. D. Birkhoff [Bir1], 1917, and [Bir2], 1927).

$$
{ }^{\#} \mathrm{CG}\left(S^{n}, g\right) \geq 1, \quad \forall g \in \mathcal{R}\left(S^{n}\right) .
$$

In 1951, L. Lyusternik and A. Fet proved the following important theorem:

Theorem 1.3 (L. Lyusternik and A. Fet [LF], 1951). For every compact manifold $M$,

$$
{ }^{\#} \mathrm{CG}(M, g) \geq 1, \quad \forall g \in \mathcal{R}(M) .
$$

Note that this theorem also holds for Finsler metrics, because the proof of Theorem 1.3 (cf. [Kli1] and [Kli2]) is variational and does not really depend on the special properties of Riemannian metrics.

Denote by $\Lambda M$ the free loop space of a Riemannian manifold $(M, g)$ and by $\Lambda^{0} M$ the single point loops on $M$. For the Finsler case, we choose a Riemannian metric on $M$, and define $\Lambda M$ similarly. In 1969, D. Gromoll and W. Meyer proved the following important result: 
Theorem 1.4 (D. Gromoll and W. Meyer [GM], 1969). Let $(M, g)$ be a Riemannian manifold such that the Betti numbers $\left\{b_{i}(\Lambda M)\right\}_{i \geq 1}$ are unbounded. Then ${ }^{\#} \mathrm{CG}(M, g)$ $=\infty$, where $b_{i}(\Lambda M)=\operatorname{rank} H_{i}\left(\Lambda M, \Lambda^{0} M ; \mathcal{K}\right)$ for all $i \in \mathbb{N}$ and some field $\mathcal{K}$.

Motivated by Theorem 1.4. M. Vigué-Poirrier and D. Sullivan proved the following remarkable result:

Theorem 1.5 (M. Vigué-Poirrier and D. Sullivan [VS], 1976). For a compact simply connected Riemannian manifold $(M, g)$, the Betti number sequence $\left\{b_{i}(\Lambda M)\right\}_{i \geq 1}$ is unbounded if and only if the cohomology algebra of $M$ requires at least two generators.

Note that in 1980, H. Matthias [Mat] generalized Theorem 1.4 to Finsler manifolds. Therefore by Theorems 1.4 and 1.5 the most interesting unsolved problem on closed geodesics is for Finsler and Riemannian spheres.

Around 1990, V. Bangert (cf. [Ban1], [BBan2]) and J. Franks (cf. [Fra1], [Fra2]) proved the following important result for Riemannian $S^{2}$ :

Theorem 1.6 (V. Bangert [Ban2], 1993, and J. Franks [Fra1], 1992).

$$
{ }^{\#} \mathrm{CG}\left(S^{2}, g\right)=\infty, \quad \forall g \in \mathcal{R}\left(S^{2}\right) .
$$

For the closed geodesic problem on Riemannian (2-dimensional) manifolds, we refer the readers to the excellent survey papers [Ban1] and [Tai].

On the other hand, in 1973 A. Katok constructed a remarkable Finsler metric on $S^{2}$ which showed that there is a major difference between Riemannian and general Finsler metrics:

Theorem 1.7 (A. Katok [Kat], 1973). For any irrational number $\alpha \in(0,1)$, there exists a Finsler metric $F_{\alpha}$ on $S^{2}$ which has precisely two distinct prime closed geodesics.

In fact, by [Kat] and W. Ziller's paper [Zil2], Katok's metric has the form $F_{\alpha}(x)=\|x\|_{g}^{*}+$ $\alpha x(V)$ for any $x \in T^{*} S^{2}$, where $\|\cdot\|_{g}$ is the standard Riemannian metric on $S^{2}$, and $V$ is a vector field corresponding to rotations on $S^{2}$ along the equatorial direction. Locally in spherical coordinates away from the north and south poles, $F_{\alpha}$ has the form

$$
F_{\alpha}\left(q_{1}, q_{2}, p_{1}, p_{2}\right)=\left(p_{1}^{2} \cos ^{-2} q_{2}+p_{2}^{2}\right)^{1 / 2}+\alpha p_{1} .
$$

The two closed geodesics on $\left(S^{2}, F_{\alpha}\right)$ are along the equator and are in fact mutually inverse curves $c$ and $c^{-1}$. They have lengths length $(c)=2 \pi /(1+\alpha)$ and length $\left(c^{-1}\right)=$ $2 \pi /(1-\alpha)$. The linearized Poincaré map $P_{c}$ of $c$ is conjugate to the rotation matrix

$$
R\left(\theta_{c}\right)=\left(\begin{array}{cc}
\cos \theta_{c} & -\sin \theta_{c} \\
\sin \theta_{c} & \cos \theta_{c}
\end{array}\right)
$$

with $\theta_{c}=2 \pi /(1+\alpha)$. Similarly $P_{c^{-1}}$ is also conjugate to $R\left(\theta_{c^{-1}}\right)$ with $\theta_{c^{-1}}=2 \pi /(1-\alpha)$. All iterates of $c$ and $c^{-1}$ are non-degenerate. Then by the precise index iteration formulae of the author proved in [Lon1], 2000, one can show that the Morse index sequences of iterates of $c$ and $c^{-1}$ counting multiplicity satisfy $\left\{i\left(c^{m}\right), i\left(c^{-m}\right)\right\}_{m \geq 1}=$ $\{1,3,3,5,5,7,7, \ldots\}$. 


\subsection{Known multiplicity results}

Based upon Katok's metric, D. Anosov asked the following question on the minimal number of closed geodesics on Finsler spheres in his 1974 ICM report [Ano]: For the ndimensional sphere $S^{n}$, Katok's example gives an irreversible Finsler metric, arbitrarily near to the 'standard' metric (to the metric of constant curvature) which has $2[n / 2]$ closed geodesics. This number coincides with the lower bound which one naturally expects for irreversible Finsler metrics on $S^{n}$ and which can be proved for metrics sufficiently near the 'standard' metric. Here $[a]=\max \{k \in \mathbb{Z} \mid k \leq a\}$ for any real number $a$. Using our notations, what Anosov expected is

$$
{ }^{\#} \mathrm{CG}\left(S^{n}, F\right) \geq 2[n / 2], \quad \forall F \in \mathcal{F}\left(S^{n}\right) .
$$

Note that in [LZ], a similar lower bound $[n / 2]+1$ on the number of closed characteristics on convex compact hypersurfaces in $\mathbb{R}^{2 n}$ is proved. We also note that in [Zil2], W. Ziller made a somewhat different conjecture for $S^{n}$ :

$$
{ }^{\#} \mathrm{CG}\left(S^{n}, F\right) \geq n, \quad \forall F \in \mathcal{F}\left(S^{n}\right) .
$$

We are only aware of a few partial answers to these conjectures for $S^{2}$.

Theorem 1.8 (H.-B. Rademacher [Rad1], 1989). Let $F$ be a bumpy Finsler metric on $S^{2}$, i.e., all the closed geodesics and their iterations on $\left(S^{2}, F\right)$ are non-degenerate. Then

$$
{ }^{\#} \mathrm{CG}\left(S^{2}, F\right) \geq 2 .
$$

In the 2003 paper [HWZ2], H. Hofer, K. Wysocki, and E. Zehnder studied Hamiltonian systems on star-shaped hypersurfaces in $\mathbb{R}^{4}$. Their result can be applied to Finsler 2 spheres to yield:

Theorem 1.9 (H. Hofer, K. Wysocki, and E. Zehnder [HWZ2], 2003). Let F be a bumpy Finsler metric on $S^{2}$. Assume that the stable and unstable manifolds at every hyperbolic closed geodesic intersect transversally. Then

$$
{ }^{\#} \mathrm{CG}\left(S^{2}, F\right)=2 \text { or } \infty .
$$

In 1993 and 1997, N. Hingston proved two theorems in [Hin1] and [Hin2] respectively which showed the existence of infinitely many prime closed geodesics on Riemannian manifolds under certain sufficient conditions. In particular, Hingston's two theorems can be adapted to Finsler 2-spheres and yield the following theorem:

Theorem 1.10 (N. Hingston [Hin1], 1993, and [Hin2], 1997). Let F be a Finsler metric on $S^{2}$ and $c$ is a closed geodesic on $\left(S^{2}, F\right)$ such that $S^{1} \cdot c^{m}$ is isolated as a critical orbit of the energy functional $E$ on $\Lambda S^{2}$ for all $m \geq 1$. Set

$$
k_{j}(c)=\operatorname{rank} H_{j}\left(N_{c}^{-} \cup\{c\}, N_{c}^{-} ; \mathbb{Q}\right) \quad \text { for } j=0,1,2,
$$

where $N_{c}$ is a slice in $\operatorname{ker} E^{\prime \prime}(c)$ transversal to $S^{1} \cdot c$, and $N_{c}^{-}=\left\{x \in N_{c} \mid E(x)<E(c)\right\}$. Suppose that either 
(i) $i\left(c^{m}\right)=m(i(c)+1)-1, v\left(c^{m}\right)=v(c)$ for all $m \geq 1$ and $k_{0}(c)>0$, or

(ii) $i\left(c^{m}\right)+v\left(c^{m}\right)=m(i(c)+v(c)-1)+1, v\left(c^{m}\right)=v(c)$ for all $m \geq 1$ and $k_{v(c)}(c)>0$.

Then ${ }^{*} \mathrm{CG}\left(S^{2}, F\right)=\infty$.

Based on the results of W. Klingenberg [Kli1], 1968, and of W. Ballmann, G. Thorbergsson, and W. Ziller [BTZ1], 1982, about closed geodesics on Riemannian spheres under pinching conditions, H.-B. Rademacher generalized those results to Finsler spheres and proved:

Theorem 1.11 (H.-B. Rademacher [ $\overline{\operatorname{Rad} 3]}$, 2005). For $F \in \mathcal{F}\left(S^{2}\right)$ let $\lambda=\max \{F(-v) \mid$ $\left.F(v)=1, v \in T S^{2}\right\}$. Suppose the flag curvature $K$ of $\left(S^{2}, F\right)$ satisfies $\lambda^{2}(\lambda+1)^{-2}<$ $\delta \leq K \leq 1$. Then

$$
{ }^{\#} \mathrm{CG}\left(S^{2}, F\right) \geq 2 .
$$

Denote the two closed geodesics by $c_{1}$ and $c_{2}$ with length $\left(c_{1}\right) \leq \operatorname{length}\left(c_{2}\right)$. Then

$$
\text { length }\left(c_{1}\right) \leq 2 \pi / \sqrt{\delta}, \quad \text { length }\left(c_{2}\right) \leq \frac{\pi}{\sqrt{\delta}}\left(\frac{\lambda}{\sqrt{\delta}(\lambda+1)-\lambda}+3\right),
$$

and $c_{1}$ is simple.

\subsection{Known stability results}

As usual, denote by $P_{c}$ the linearized Poincaré map of a closed geodesic $c$ on a manifold $M$ and $\mathbb{U}=\{z \in \mathbb{C}|| z \mid=1\}$. Then $c$ is hyperbolic if $\sigma\left(P_{c}\right) \cap \mathbb{U}=\emptyset$, elliptic if $\sigma\left(P_{c}\right) \subset \mathbb{U}$, and non-degenerate if $1 \notin \sigma\left(P_{c}\right)$. Note that $P_{c^{m}}=P_{c}^{m}$ for all $m \geq 1$. For a closed geodesic $c$ on a 2-dimensional surface, $c$ is irrationally elliptic or rationally elliptic if $P_{c}$ is conjugate to a rotation matrix 11.1 with $\theta_{c} / \pi \in \mathbb{R} \backslash \mathbb{Q}$ or $\theta_{c} / \pi \in \mathbb{Q}$ respectively,

In 1982-83, W. Ballmann, G. Thorbergsson, and W. Ziller studied the stability of closed geodesics on $S^{n}$; specifically, they proved:

Theorem 1.12 (W. Ballmann, G. Thorbergsson, and W. Ziller [BTZ2], 1983). For $g \in$ $\mathcal{R}\left(S^{n}\right)$, if the sectional curvature satisfies $4 / 9 \leq K \leq 1$, then there exist at least two elliptic closed geodesics on $\left(S^{n}, g\right)$.

In 1989 and 2005, H.-B. Rademacher studied Finsler spheres $S^{n}$, and proved

Theorem 1.13 (H.-B. Rademacher [Rad1], 1989). Let $F \in \mathcal{F}\left(S^{n}\right)$ be bumpy and satisfy ${ }^{\#} \mathrm{CG}\left(S^{2}, F\right)<\infty$. Then there exists at least two irrationally elliptic closed geodesics on $\left(S^{2}, F\right)$.

Theorem 1.14 (H.-B. Rademacher [Rad3], 2005). Let $F \in \mathcal{F}\left(S^{n}\right)$ satisfy

$$
\lambda<2, \quad\left(\frac{3 \lambda}{2(\lambda+1)}\right)^{2}<\delta \leq K \leq 1
$$

where $\lambda$ is defined in Theorem 1.11. Then there exists at least one elliptic closed geodesic on $\left(S^{n}, F\right)$. 
Note that in 2000 the author proved a related stability result for closed characteristics on convex compact hypersurfaces in $\mathbb{R}^{4}$ :

Theorem 1.15 (Y. Long [Lon1], 2000). Let $\Sigma$ be a convex compact $C^{2}$ hypersurface in $\mathbb{R}^{4}$ with precisely two closed characteristics on $\Sigma$. Then both of them are elliptic.

\section{Main new multiplicity and stability results}

Recently, V. Bangert and the author proved the following result which settles Anosov's conjecture 1.2 as well as 1.3 for $S^{2}$ positively. This theorem was first reported in July 2004 at the conference to celebrate Professor I. Ekeland's 60th birthday.

Theorem 2.1 (V. Bangert and Y. Long [BL], 2005).

$$
{ }^{\#} \mathrm{CG}\left(S^{n}, F\right) \geq 2, \quad \forall F \in \mathcal{F}\left(S^{2}\right) .
$$

Combining the result in [HWZ1] of H. Hofer, K. Wysocki and E. Zehnder, 1998, with Theorems 1.9 and 2.1, it is natural to make the following conjecture:

Conjecture 1. We have

$$
{ }^{\#} \mathrm{CG}\left(S^{2}, F\right)=2 \text { or } \infty, \quad \forall F \in \mathcal{F}\left(S^{2}\right) .
$$

Motivated by Theorem 2.1 and those mentioned in Subsections 1.2, recently my Ph.D. student Wei Wang and myself proved the following result:

Theorem 2.2 (Y. Long and W. Wang [LW], 2005). Let $F \in \mathcal{F}\left(S^{2}\right)$ satisfy ${ }^{\#} \mathrm{CG}\left(S^{2}, F\right)$ $<\infty$. Then there exists at least two irrationally elliptic closed geodesics on $\left(S^{2}, F\right)$.

As a consequence we obtain

Corollary 2.3 (Y. Long and W. Wang [LW], 2005). Let $F \in \mathcal{F}\left(S^{2}\right)$ satisfy ${ }^{\#} \mathrm{CG}\left(S^{2}, F\right)$ $=2$. Then the two closed geodesics $c_{1}$ and $c_{2}$ on $\left(S^{2}, F\right)$ are both irrationally elliptic with rotation angles $\theta_{1}=2 \pi /(1+\alpha)$ and $\theta_{2}=2 \pi /(1-\alpha)$ respectively for some $\alpha \in(0,1) \backslash \mathbb{Q}$.

It is rather surprising that if Conjecture 1 holds, then Corollary 2.3 implies that whenever ${ }^{\#} \mathrm{CG}\left(S^{2}, F\right)$ is finite, there are precisely two distinct prime irrationally elliptic closed geodesics and they behave analytically like those two prime closed geodesics of Katok's metric, i.e., whose iterations possess the same Morse indices and nullities. Then their local critical modules are all the same as the two of Katok. Note that here these two prime closed geodesics may not be inverse curves to each other as are Katok's.

\section{Main ideas in the proof of Theorem 2.1}

The conditions (F1)-(F3) for Finsler metrics were introduced by P. Finsler for the local existence and uniqueness of geodesics connecting two nearby points on a manifold. The problem of closed geodesics is global. Thus our proof of Theorem 2.1 is naturally topo- 
logical and variational, and does not depend on geometrical properties of each individual Finsler metric such as curvatures.

The main ideas in the proof of Theorem 2.1 of V. Bangert and the author are contained in the following four steps, where we explain more on the rationally elliptic case. Here to make explanations shorter, topics related to the smoothness of the energy functional $E$ on the free loop space on $S^{2}$ are all omitted. We concentrate on topological facts and variational arguments which are related to multiplicity.

Fix an $F \in \mathcal{F}\left(S^{2}\right)$. Assuming that there exists precisely one prime closed geodesic $c$ on $\left(S^{2}, F\right)$, we proceed as follows to reach a contradiction. In this section we use homology modules with $\mathbb{Q}$-coefficients only.

〈1〉 By the author's precise index iteration formulae proved in Section 3 of [Lon1], 2000 (cf. Section 8.1 of [Lon2]), there are nine possibilities for the closed geodesic $c$ depending on the eigenvalues of the linearized Poincaré map $P_{c}$ which is a $2 \times 2$ symplectic matrix. Here the first three cases are for eigenvalue 1, the next three cases are for eigenvalue -1 , the 7 th is for the rationally elliptic case, the 8 th is for the irrationally elliptic case, and the 9th is for the hyperbolic case. Note that the closed geodesic $c$ and all of its iterates are non-degenerate in the last two cases in which two closed geodesics were found by H.-B. Rademacher [Rad1], 1989. Therefore we only need to study the first seven degenerate cases.

Here we choose a Riemannian metric $g$ on $S^{2}$, and define $\Lambda=\Lambda S^{2}$ to be the free loop space of $H^{1}\left(S^{1}, S^{2}\right)$, where a curve $c$ is $H^{1}$ if it is absolutely continuous and $\dot{c}(t)$ is square integrable in $g$ as in Chapter 1 of [Kli2].

Define $E(c)=\int_{0}^{1} F^{2}(\dot{c}(t)) d t$ and $\Lambda^{a}=\{\gamma \in \Lambda \mid E(\gamma) \leq a\}$ for $a \in \mathbb{R}$.

$\langle 2\rangle$ In order to apply Morse theory, using the arguments of W. Ziller [Zil1], 1977, we obtain the Betti numbers as follows (cf. V. Bangert and Y. Long [BL], 2005):

$$
\begin{array}{r}
H_{q}\left(\Lambda, \Lambda^{0}\right)=\left\{\begin{array}{lll}
0 & \text { if } q \leq 0 & \text { or } q=2, \\
\mathbb{Q} & \text { if } q=1 \text { or } q \geq 3 .
\end{array}\right. \\
b_{q} \equiv \operatorname{rank} H_{q}\left(\Lambda, \Lambda^{0}\right)=\left\{\begin{array}{lll}
0 & \text { if } q \leq 0 \text { or } q=2, \\
1 & \text { if } q=1 \text { or } q \geq 3 .
\end{array}\right.
\end{array}
$$

As usual the Morse type number $M_{k}$ for all $k \geq 0$ is defined by

$$
M_{k}=\sum_{\substack{1 \leq j \leq q \\ m \geq 1}} \operatorname{rank} H_{k}\left(\Lambda\left(c^{m}\right) \cup\left\{S^{1} \cdot c^{m}\right\}, \Lambda\left(c^{m}\right)\right), \quad \forall k \geq 0,
$$

where $\Lambda\left(c^{m}\right)=\left\{\gamma \in \Lambda \mid E(\gamma)<E\left(c^{m}\right)\right\}$. Then for every integer $k \geq 1$, it is well known that the following Morse inequalities hold:

$$
\begin{gathered}
M_{k} \geq b_{k}, \\
M_{k}-M_{k-1}+M_{k-2}-\cdots+(-1)^{k-1} M_{1}+(-1)^{k} M_{0} \\
\geq b_{k}-b_{k-1}+b_{k-2}-\cdots+(-1)^{k-1} b_{1} .
\end{gathered}
$$

$\langle 3\rangle$ For each of the first six cases, using index iteration formulae of [Lon1] we obtain precisely all the Morse indices and nullities of all iterations $c^{m}$ of $c$. Applying also the 
techniques of D. Gromoll and W. Meyer [GM], 1969, we are able to compute all the local critical modules of the energy functional $E(c)=\int_{0}^{1} F^{2}(\dot{c}(t)) d t$ near $c^{m}$ for all $m \geq 1$ in $\Lambda$. Then we find that either the Morse inequalities already yield a contradiction which implies the existence of at least two distinct prime closed geodesics on $\left(S^{2}, F\right)$, or the Morse inequalities lift up the dimension of a certain local homology group. Then Hingston's Theorem 1.10 becomes applicable and yields infinitely many distinct prime closed geodesics on $\left(S^{2}, F\right)$, which completes the proof for this case.

For example, when $P_{c}=-I$, by the Morse inequality $M_{1} \geq b_{1}$, we obtain $i(c)=1$ and $v(c)=0$. By Theorem 8.1 .5 of [Lon2], we obtain

$$
i\left(c^{m}\right)=m-\frac{1+(-1)^{m}}{2}, \quad v\left(c^{m}\right)=1+(-1)^{m}, \quad \forall m \geq 1 .
$$

We set

$$
\hat{k}_{j}\left(c^{m}\right)=\operatorname{rank} H_{j}\left(N_{c^{m}}^{-} \cup\left\{c^{m}\right\}, N_{c^{m}}^{-}\right)^{\mathbb{Z}_{m}} \quad \text { for } j=0,1, \ldots, v\left(c^{m}\right) .
$$

Note that

$$
\hat{k}_{j}\left(c^{m}\right) \leq k_{j}\left(c^{m}\right), \quad \forall m \geq 1 .
$$

Using the method of D. Gromoll and W. Meyer [GM], all the local critical modules can be computed and we obtain

$M_{0}=0, M_{1}=1+\hat{k}_{0}\left(c^{2}\right), \quad M_{2}=1+\hat{k}_{0}\left(c^{2}\right)+\hat{k}_{1}\left(c^{2}\right), \quad M_{3}=1+\hat{k}_{1}\left(c^{2}\right)+\hat{k}_{2}\left(c^{2}\right)$.

Therefore by the Morse inequality we obtain

$$
1+k_{2}\left(c^{2}\right) \geq 1+\hat{k}_{2}\left(c^{2}\right)=M_{3}-M_{2}+M_{1} \geq b_{3}-b_{2}+b_{1}=2 .
$$

Let $d=c^{2}$. Then 3.5 and 3.6 yield condition (ii) of Theorem 1.10 and thus ${ }^{\#} \mathrm{CG}\left(S^{2}, F\right)$ $=\infty$.

$\langle 4\rangle$ For the 7th case, $P_{c}$ is conjugate to the matrix $R(\theta)$ of $(1.1)$ for some rotation angle $\theta \in(0,2 \pi) \cap(\pi \mathbb{Q} \backslash\{\pi\})$, i.e., $c$ is rationally elliptic.

By the Morse inequality $M_{1} \geq b_{1}=1$, we obtain $i(c)=1$. Thus by Theorem 8.1.7 of [Lon2],

$$
\begin{array}{ll}
i\left(c^{m}\right)=2\left[\frac{m \theta}{2 \pi}\right]+1, \quad v\left(c^{m}\right)=0, & \text { if } m \theta \neq 0 \bmod 2 \pi, \\
i\left(c^{m}\right)=2\left[\frac{m \theta}{2 \pi}\right]-1, \quad v\left(c^{m}\right)=2, & \text { if } m \theta=0 \bmod 2 \pi .
\end{array}
$$

Therefore there is a unique minimal integer $n \geq 3$ such that $v\left(c^{n}\right)=2$.

By (3.8), both the iteration formulae in (i) and (ii) of Theorem 1.10 hold for the iterates of $c^{n}$. Thus we obtain ${ }^{\#} \mathrm{CG}\left(S^{2}, F\right)=\infty$ by Theorem 1.10 whenever $k_{0}\left(c^{n}\right)+k_{2}\left(c^{n}\right)>0$. Therefore we need only study case 7 under the condition

$$
k_{0}\left(c^{n}\right)=k_{2}\left(c^{n}\right)=0 .
$$


Let $\kappa_{m}=E\left(c^{m}\right)$ for all $m \geq 1$. Then $\kappa_{m} \rightarrow \infty$ as $m \rightarrow \infty$ and

$$
0<\kappa_{1}<\kappa_{2}<\cdots<\kappa_{i}<\kappa_{i+1}<\cdots .
$$

As an example we consider the case of $\theta=\pi / 3, n=6$, and $k_{0}\left(c^{6}\right)=k_{1}\left(c^{6}\right)=$ $k_{2}\left(c^{6}\right)=0$. Then we obtain $M_{k}=5$ for all $k \geq 1$. Thus the left hand side of the Morse inequality (3.4) is always 5 and the right hand side of (3.4) is at most 2. Therefore (3.4) always holds and the Morse inequality yields no information. Likewise Hingston's Theorem 1.10 does not apply because $k_{0}\left(c^{6}\right)=k_{2}\left(c^{6}\right)=0$. Thus this case requires some new ideas and a new approach. Here we make the following comparison of long exact sequences for the triple $\left(\Lambda, \Lambda^{\kappa_{\tau}}, \Lambda^{0}\right)$ :

$$
\begin{array}{ccccc}
0 & 0 & \mathbb{Q} & \mathbb{Q} & 0 \\
\| & \| & \| & \| & \| \\
H_{2}\left(\Lambda, \Lambda^{0}\right) \rightarrow H_{2}\left(\Lambda, \Lambda^{\kappa_{\tau}}\right) & \rightarrow & H_{1}\left(\Lambda^{\kappa_{\tau}}, \Lambda^{0}\right) & \rightarrow & H_{1}\left(\Lambda, \Lambda^{0}\right) \\
\| & \| & H_{1}\left(\Lambda, \Lambda^{\kappa_{\tau}}\right) \\
0 & 0 & \mathbb{Q}^{\tau} & \| & \|
\end{array}
$$

Here the top line gives the case of Katok's example with $\tau=1$, which matches up perfectly. The bottom line is for our sample of $\theta=\pi / 3$ with $\tau=5$, which yields a contradiction. This comparison yields an important idea for dealing with the general case. Here our crucial observation is that the alternative summation in the Morse inequality indicates how the higher dimensional local critical groups kill the lower dimensional local critical groups at the dimension level. This leads to a rather rough understanding of the mutual relations among these local homology groups. To understand them further we need to study them more carefully at the homological level.

For the general case, let $\tau=\max \{j \geq 1 \mid j \theta<2 \pi\}$. Then we have the following three important claims:

Claim 1. $2 \leq \tau \leq n-1$.

Claim 2. $H_{1}\left(\Lambda^{\kappa_{\tau}}, \Lambda^{0}\right)=\bigoplus_{m=1}^{\tau} H_{1}\left(\Lambda^{\kappa_{m}}, \Lambda^{\kappa_{m-1}}\right)=\mathbb{Q}^{\tau}$.

Claim 3. $H_{2}\left(\Lambda, \Lambda^{\kappa_{\tau}}\right)=0$ when $\tau<n-1$, while $H_{2}\left(\Lambda, \Lambda^{\kappa_{\tau}}\right)=\mathbb{Q}^{a}$ for some $a \in$ $[0, n-3]$ when $\tau=n-1$.

Assuming these three claims for the moment, we continue our study of case 7 under the condition 3.9. As suggested by 3.10) we consider the long exact sequence for the triple $\left(\Lambda, \Lambda^{\kappa_{\tau}}, \Lambda^{0}\right)$. By 3.2 and Gromoll-Meyer's technique in [GM] for computing local homology modules we obtain

$$
\begin{aligned}
& H_{2}\left(\Lambda, \Lambda^{0}\right) \rightarrow H_{2}\left(\Lambda, \Lambda^{\kappa_{\tau}}\right) \rightarrow H_{1}\left(\Lambda^{\kappa_{\tau}}, \Lambda^{0}\right) \rightarrow H_{1}\left(\Lambda, \Lambda^{0}\right) \rightarrow H_{1}\left(\Lambda, \Lambda^{\kappa_{\tau}}\right) \\
& \begin{array}{lcccc}
\| & \| & \| & \| & \| \\
0 & \mathcal{H}_{2} & \mathbb{Q}^{\tau} & \mathbb{Q} & 0
\end{array}
\end{aligned}
$$

When $\tau<n-1$, we have $\mathcal{H}_{2}=0$ by Claim 3. Thus (3.11) yields

$$
\mathbb{Q}^{\tau}=0 \oplus \mathbb{Q}=\mathbb{Q} \text {. }
$$

This contradicts the fact that $\tau \geq 2$ by Claim 1 . 
When $\tau=n-1$, we have $\mathcal{H}_{2}=\mathbb{Q}^{a}$ for some $a \in[0, n-3]$ by Claim 3. Thus 3.11 yields

$$
\mathbb{Q}^{n-1}=\mathbb{Q}^{\tau}=\mathbb{Q}^{a} \oplus \mathbb{Q}=\mathbb{Q}^{a+1} .
$$

This contradicts the fact that $a \leq n-3$.

Therefore we are reduced to proving Claims 1 to 3 .

To prove Claim 1, we use the condition (3.9) and an identity satisfied by the mean index $\hat{i}(c) \equiv \lim _{m \rightarrow \infty} i\left(c^{m}\right) / m=\theta / \pi$ of $c$, and derive an important estimate $0<\theta<\pi$. It implies Claim 1.

In general the homology groups on different level sets may not be additive. We are only aware of two papers: R. Bott and H. Samelson [BS], 1958, and W. Ziller [Zil1], 1977, who studied such homological addition properties of level sets in the loop spaces for compact globally symmetric spaces. But our $\left(S^{2}, F\right)$ is not a globally symmetric space in general and their techniques do not apply. For the proof of Claim 2, we carry out precise computations on the connecting homomorphisms between level sets and prove the following vanishing property in the long exact sequence for the triple $\left(\Lambda^{\kappa_{m}}, \Lambda^{\kappa_{m-1}}, \Lambda^{0}\right)$ :

$$
\partial_{2}=0: H_{2}\left(\Lambda^{\kappa_{m}}, \Lambda^{\kappa_{m-1}}\right) \rightarrow H_{1}\left(\Lambda^{\kappa_{m-1}}, \Lambda^{0}\right) .
$$

Here the precise relations 3.7 and 3.8 for the Morse indices and nullities of $c^{m}$ with $1 \leq m \leq \tau$ are crucial. This yields

$$
H_{1}\left(\Lambda^{\kappa_{m}}, \Lambda^{0}\right)=H_{1}\left(\Lambda^{\kappa_{m-1}}, \Lambda^{0}\right) \oplus H_{1}\left(\Lambda^{\kappa_{m}}, \Lambda^{\kappa_{m-1}}\right), \quad \forall m=1, \ldots, \tau,
$$

which yields Claim 2 .

When $\tau<n-1$, by direct computation we obtain $\mathcal{H}_{2}=0$ in 3.11.

When $\tau=n-1$, together with the mean index identity mentioned above we obtain $2 \pi=n \theta=\left(n-1-\hat{k}_{1}\left(c^{n}\right)\right) \pi$, which implies $\hat{k}_{1}\left(c^{n}\right)=n-3$. By the long exact sequence for the triple $\left(\Lambda, \Lambda^{\kappa_{\tau+1}}, \Lambda^{\kappa_{\tau}}\right)$ we obtain

$$
\mathbb{Q}^{\hat{k}_{1}\left(c^{n}\right)}=H_{2}\left(\Lambda^{\kappa_{\tau+1}}, \Lambda^{\kappa_{\tau}}\right) \rightarrow H_{2}\left(\Lambda, \Lambda^{\kappa_{\tau}}\right) \equiv \mathcal{H}_{2} \rightarrow H_{2}\left(\Lambda, \Lambda^{\kappa_{\tau+1}}\right)=0 .
$$

Therefore Claim 3 holds.

This completes our study of case 7 and the proof of Theorem 2.1

\section{Open problems}

Besides Conjecture 1 mentioned in Section 2 and the Anosov Conjecture (1.2), I believe that the following further problems are interesting and important for further studies of the closed geodesic problem on Finsler as well as Riemannian spheres.

Conjecture 2. For every $F \in \mathcal{F}\left(S^{n}\right)$, there exist two integers $2 \leq p_{n} \leq q_{n}$ such that

$$
\left\{{ }^{\#} \mathrm{CG}\left(S^{n}, F\right) \mid F \in \mathcal{F}\left(S^{n}\right)\right\}=\left\{k \in \mathbb{N} \mid p_{n} \leq k \leq q_{n}\right\} \cup\{\infty\} .
$$

More specifically, we suspect that 
Conjecture 3. $\left\{{ }^{\#} \mathrm{CG}\left(S^{3}, F\right) \mid F \in \mathcal{F}\left(S^{3}\right)\right\}=\{2,3,4\} \cup\{\infty\}$.

For this conjecture, very little is known apart from the case of Katok's metric $F$ which satisfies ${ }^{\#} \mathrm{CG}\left(S^{3}, F\right)=4$ (cf. [Kat] and [Zil2]).

Conjecture 4. ${ }^{\#} \mathrm{CG}\left(S^{n}, g\right)=\infty$ for every $g \in \mathcal{R}\left(S^{n}\right)$ with $n \geq 3$.

Note that so far it seems to be unknown whether ${ }^{\#} \operatorname{CG}\left(S^{n}, g\right) \geq 2$ for all $g \in \mathcal{R}\left(S^{n}\right)$ when $n \geq 3$.

Conjecture 5. There exists at least one elliptic closed geodesic on $\left(S^{n}, F\right)$ for every $F \in \mathcal{F}\left(S^{n}\right)$.

For $S^{2}$, by our Theorem 2.2, it is only necessary to study the case when ${ }^{\#} \mathrm{CG}\left(S^{2}, F\right)=\infty$ for which it seems unfortunately that there is no effective method available yet without pinching conditions. On the other hand, in an interesting paper [Grj], 1980, A. Grjuntal' proved the existence of Riemannian metrics on $S^{2}$ with positive curvature whose closed non-selfintersecting geodesics are all hyperbolic.

Because our proofs of Theorems 2.1 and 2.2 are variational, we hope that they may help at least in the study of some of the above conjectures for Finsler (as well as Riemannian) spheres.

Acknowledgments. The author thanks sincerely Professor Victor Bangert for helpful discussions on related problems. He also thanks Professors Vittorio Coti Zelati, Mario Girardi, and Patrizia Pucci for inviting him to join the conference in Rome in January 2005 on the occasion of the 60th birthday of Professor Antonio Ambrosetti.

\section{References}

[Ano] Anosov, D. V.: Geodesics in Finsler geometry. In: Proc. I.C.M. (Vancouver, B.C., 1974), Vol. 2, Montreal, 293-297 (1975) (in Russian); Amer. Math. Soc. Transl. 109, 81-85 (1977) Zbl 0368.53045

[BTZ1] Ballmann, W., Thorbergsson, G., Ziller, W.: Closed geodesics on positively curved manifolds. Ann. of Math. 116, 213-247 (1982) Zbl 0495.58010 MR 0672836

[BTZ2] Ballmann, W., Thorbergsson, G., Ziller, W.: Existense of closed geodesics on positively curved manifolds. J. Differential Geom. 18, 221-252 (1983) Zbl 0514.53044 MR 0710053

[Ban1] Bangert, V.: Geodätische Linien auf Riemannschen Mannigfaltigkeiten. Jber. Deutsch. Math.-Verein. 87, 39-66 (1985) Zbl 0565.53028 MR 0789708

[Ban2] Bangert, V.: On the existence of closed geodesics on two-spheres. Internat. J. Math. 4, 1-10 (1993) Zbl 0791.53048 MR 1209957

[BL] Bangert, V., Long, Y.: The existense of two closed geodesics on every Finsler 2-sphere. Preprint (2005)

[BCS] Bao, D., Chern, S. S., Shen, Z.: An Introduction to Riemann-Finsler Geometry. Springer. Berlin (2000) Zbl 0954.53001 MR 1747675

[Bir1] Birkhoff, G. D.: Dynamical systems with two degrees of freedom. Trans. Amer. Math. Soc. 18, 199-300 (1917) JFM 46.1174.01 MR 1501070 
[Bir2] Birkhoff, G. D.: Dynamical Systems. Amer. Math. Soc. Colloq. Publ. 9, Amer. Math. Soc., Providence, RI (1927) JFM 53.0732.01

[BS] Bott, R., Samelson, H.: Applications of the theory of Morse to symmetric spaces. Amer. J. Math. 80, 964-1029 (1958) Zbl 0101.39702 MR 0105694

[Cha] Chang, K. C.: Infinite Dimensional Morse Theory and Multiple Solution Problems. Birkhäuser, Boston (1993) Zbl 0779.58005 MR 1196690

[Fra1] Franks, J.: Geodesics on $S^{2}$ and periodic points of annulus diffeomorphisms. Invent. Math. 108, 403-418 (1992) Zbl 0766.53037 MR 1161099

[Fra2] Franks, J.: Area preserving homeomorphisms of open surfaces of genus zero. New York J. Math. 2, 1-19 (1996) Zbl 0891.58033 MR 1371312

[Grj] Grjuntal', A. I.: The existence of convex spherical metrics, all closed nonselfintersecting geodesics of which are hyperbolic. Math. USSR-Izv. 14, no. 1, 1-16 (1980) Zbl 0433.53032

[GM] Gromoll, D., Meyer, W.: Periodic geodesics on compact Riemannian manifolds. J. Differential Geom. 3, 493-510 (1969) Zbl 0203.54401 MR 0264551

[Had] Hadamard, J.: Les surfaces à courbures opposées et leurs lignes géodésiques. J. Math. Pures Appl. (5) 4, 27-73 (1898) JFM 29.0522.01

[Hin1] Hingston, N.: On the growth of the number of closed geodesics on the two-sphere. Int. Math. Res. Not. 1993, no. 9, 253-262 Zbl 0809.53053 MR 1240637

[Hin2] Hingston, N.: On the length of closed geodesics on a two-sphere. Proc. Amer. Math. Soc. 125, 3099-3106 (1997) Zbl 0889.58026 MR 1443831

[HWZ1] Hofer, H., Wysocki, K., Zehnder, E., The dynamics on three-dimensional strictly convex energy surfaces. Ann. of Math. 148, 197-289 (1998) Zbl 0944.37031 MR 1652928

[HWZ2] Hofer, H., Wysocki, K., Zehnder, E.: Finite energy foliations of tight three spheres and Hamiltonian dynamics. Ann. of Math. 157, 125-255 (2003) Zbl pre01933157 MR 1954266

[Kat] Katok, A. B.: Ergodic perturbations of degenerate integrable Hamiltonian systems. Izv. Akad. Nauk SSSR 37 (1973), 539-576 (in Russian); English transl.: Math. USSR-Izv. 7, 535-571 (1973) Zbl 0316.58010 MR 0331425

[Kli1] Klingenberg, W.: Closed geodesics. Ann. of Math. 89, 68-91 (1969) Zbl 0172.46503 MR 0239624

[Kli2] Klingenberg, W.: Lectures on Closed Geodesics. Springer, Berlin (1978) Zbl 0397.58018 MR 0478069

[Kli3] Klingenberg, W.: Riemannian Geometry. de Gruyter, Berlin; 1st ed. (1982) Zbl 0495.53036 MR 0666697, 2nd ed. (1995) Zbl 0911.53022 MR 1330918

[Lon1] Long, Y.: Precise iteration formulae of the Maslov-type index theory and ellipticity of closed characteristics. Adv. in Math. 154, 76-131 (2000) Zbl 0970.37013 MR 1780096

[Lon2] Long, Y.: Index Theory for Symplectic Paths with Applications. Progr. Math. 207, Birkhäuser, Basel (2002) Zbl 1012.37012 MR 1898560

[LW] Long, Y., Wang, W.: Stability of closed geodesics on Finsler 2-spheres. Preprint (2005)

[LZ] Long, Y., Zhu, C.: Closed characteristics on compact convex hypersurfaces in $\mathbb{R}^{2 n}$. Ann. of Math. 155, 317-368 (2002) Zbl 1028.53003 MR 1906590

[LF] Lyusternik, L. A., Fet, A. I.: Variational problems on closed manifolds. Dokl. Akad. Nauk SSSR (N.S.) 81, 17-18 (1951) (in Russian) MR 0044760

[LuS] Lyusternik, L. A., Schnirelmann, L.: Sur le problème de trois géodésiques fermées sur les surfaces de genre 0. C. R. Acad. Sci. Paris 189, 269-271 (1929)

[Mat] Matthias, H.: Zwei Verallgemeinerungen eines Satzes von Gromoll und Meyer. Bonner Math. Schriften 126 (1980) Zbl 0481.53042 MR 0598815 
[Mor] Morse, M.: Calculus of Variations in the Large. Amer. Math. Soc. Colloq. Publ. 18, Amer. Math. Soc., Providence, RI (1934) Zbl 0011.02802 MR 1451874

[Poi] Poincaré, H.: Sur les lignes géodésiques des surfaces convexes. Trans. Amer. Math. Soc. 6, 237-274 (1905) JFM 36.0669.01 MR 1500710

[Rad1] Rademacher, H.-B.: On the average indices of closed geodesics. J. Differential Geom. 29, 65-83 (1989) Zbl 0658.53042 MR 0978076

[Rad2] Rademacher, H.-B.: Morse Theorie und geschlossene Geodätische. Bonner Math. Schriften 229 (1992) Zbl 0826.58012 MR 1237191

[Rad3] Rademacher, H.-B.: Existence of closed geodesics on positively curved Finsler manifolds. Preprint (2005).

[She] Shen, Z.: Lectures on Finsler Geometry. World Sci., Singapore (2001) Zbl 0974.53002 MR 1845637

[Tai] Taumanov, I. A.: Closed extremals on two-dimensional manifolds. Russian Math. Surveys 47, no. 2, 163-211 (1992) Zbl 0791.58026 MR 1185286

[VS] Vigué-Poirrier, M., Sullivan, D.: The homology theory of the closed geodesic problem. J. Differential Geom. 11, 633-644 (1976) Zbl 0361.53058 MR 0455028

[Yau] Yau, S. T.: Problem Section. In: Seminar on Differential Geometry, S. T. Yau (ed.), Princeton Univ. Press, Princeton, 669-706 (1982) Zbl 0479.53001 MR 0645762

[Zil1] Ziller, W.: The free loop space of globally symmetric spaces. Invent. Math. 41, 1-22 (1977) Zbl 0338.58007 MR 0649625

[Zil2] Ziller, W.: Geometry of the Katok examples. Ergodic Theory Dynam. Systems 3, 135157 (1982) Zbl 0559.58027 MR 0743032 